\title{
Self-monitoring urinary salt excretion in adults: A novel education program for restricting dietary salt intake
}

\author{
KENICHIRO YASUTAKE $^{1}$, KAYOKO SAWANO $^{1}$, SHOKO YAMAGUCHI $^{1}$, \\ HIROKO SAKAI $^{2}$, HATSUMI AMADERA ${ }^{2}$ and TAKUYA TSUCHIHASHI ${ }^{3}$ \\ ${ }^{1}$ Department of Health and Nutrition Science, Faculty of Health and Social Welfare Sciences, Nishikyushu University, \\ Kanzaki; ${ }^{2}$ Department of Health Promotion, Tosu Health Center, Saga; ${ }^{3}$ Division of Hypertension, \\ Kyushu Medical Center, National Hospital Organization, Fukuoka, Japan
}

Received March 29, 2011; Accepted May 10, 2011

DOI: $10.3892 / \mathrm{etm} .2011 .265$

\begin{abstract}
This study aimed to examine the usefulness of the self-monitoring of urinary salt excretion for educating individuals about the risk of excessive dietary salt intake. The subjects were 30 volunteers (15 men and 15 women) not consuming anti-hypertensive medication. The subjects measured urinary salt excretion at home for 4 weeks using a self-monitoring device. Blood pressure (BP), anthropometric variables and nutritional variables (by a dietary-habits questionnaire) were measured before and after the measurement of urinary salt excretion. Statistical analyses were performed, including paired t-tests, Chi-square test, Pearson's product moment correlation coefficient and multiple linear regression analysis. In all subjects, the average urinary salt excretion over 4 weeks was $8.05 \pm 1.61 \mathrm{~g} /$ day and the range (maximum-minimum value) was $5.58 \pm 2.15 \mathrm{~g} /$ day. Salt excretion decreased significantly in weeks 3 and $4(\mathrm{P}<0.05$ and $\mathrm{P}<0.01$, respectively). Diastolic BP decreased from 77.7 \pm 14.3 (at baseline) to $74.3 \pm 13.3$ after 4 weeks $(\mathrm{P}<0.05)$, while systolic BP and anthropometric variables remained unchanged. Nutrition surveys indicated that energy intake was correlated with salt intake both before and after the measurements; changes in both variables during the observation period were correlated $(r=0.40, \mathrm{P}<0.05)$. The percentage of subjects who were aware of the restriction in dietary salt intake increased from 47 to $90 \%$. In conclusion, daily monitoring of the amount of urinary salt excretion using a selfmonitoring device appears to be an effective educational tool for improving the quality of life of healthy adults.
\end{abstract}

\section{Introduction}

The number of hypertensive subjects in Japan is estimated to be 40 million. Since the proportion of elderly people in the

Correspondence to: Dr Kenichiro Yasutake, Department of Health and Nutrition Science, Faculty of Health and Social Welfare Sciences, Nishikyushu University, 4490-9 Kanzaki, Saga 842-8585, Japan E-mail: yasutakekenichiro@gmail.com

Key words: urinary salt excretion, hypertension prevention, salt restriction education, self-monitoring, blood pressure population is increasing and the incidence of hypertension increases with age, management of hypertension is an important problem in our country. Lifestyle factors, particularly excess dietary salt intake, play major roles in the onset as well as the development of hypertension (1-5). Salt intake of the Japanese population was among the highest for countries that participated in the INTERSALT study (6), and thus salt restriction is the most important lifestyle modification for the Japanese.

Hypertension prevention is one of the important outcomes of 'Healthy Japan 21', which is a project designed to create a healthy nation in the 21 st century.

In 'Healthy Japan 21', the goal is a daily salt intake of less than $10 \mathrm{~g}$ in males and less than $8 \mathrm{~g}$ in females. However, according to this interim report, the goal seems to be very difficult to achieve $(7,8)$. Thus, it is important to develop strategies for educating people about the need to reduce their salt intake.

One strategy is the self-monitoring of urinary salt excretion; this was recommended as a useful strategy by the Working Group for Dietary Salt Reduction of the Japanese Society of Hypertension (9). A device that may be useful with this strategy is a self-monitoring device that was recently developed and estimates 24-h urinary salt excretion from overnight urine samples (10). However, the usefulness of this medical device as an educational tool has not yet been established. The purpose of this study, therefore, was to determine the usefulness of this urinary salt self-monitoring device for educating healthy adults regarding their levels of salt intake and the dangers of excessive salt use.

\section{Materials and methods}

Study protocol. The study protocol was approved by the Ethics Committee of Nishikyushu University. Informed written consent was obtained from each subject, and the study protocol conformed to the ethical guidelines of the 1975 Declaration of Helsinki. We prospectively enrolled healthy adult volunteers between March and April of 2009 in Saga, Japan. Exclusion criteria included physical, psychic and social difficulties, subjects under medical treatment and those $\geq 65$ years of age. A total of 30 subjects (15 males and 15 females) were enrolled in the study. 


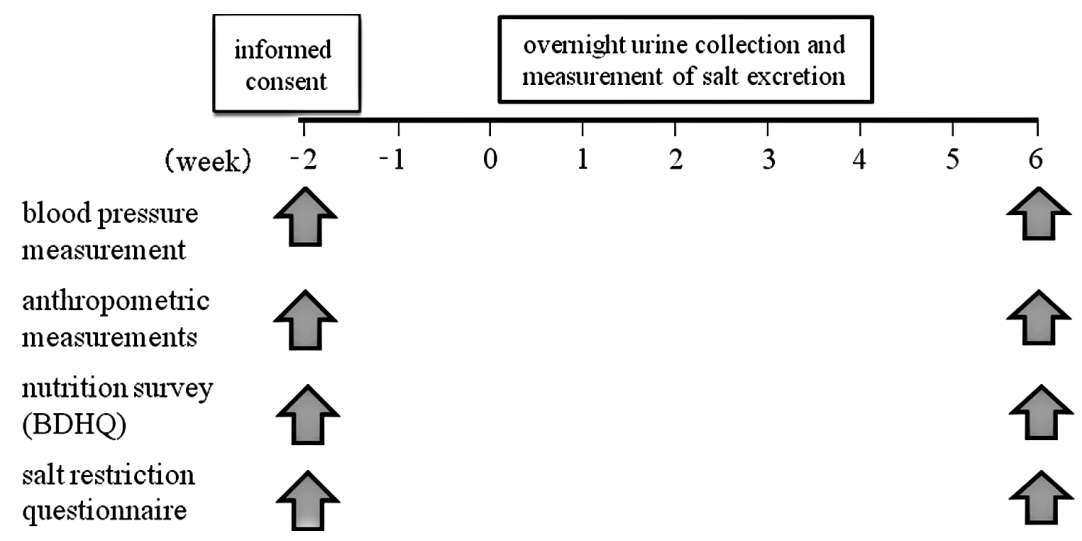

Figure 1. Study protocol. Variables were measured at baseline and 8 weeks later. Variables included blood pressure, dietary habits and nutritional parameters. Subjects collected overnight urine samples for 4 weeks. A medical device was used to measure salt excretion in the urine samples.

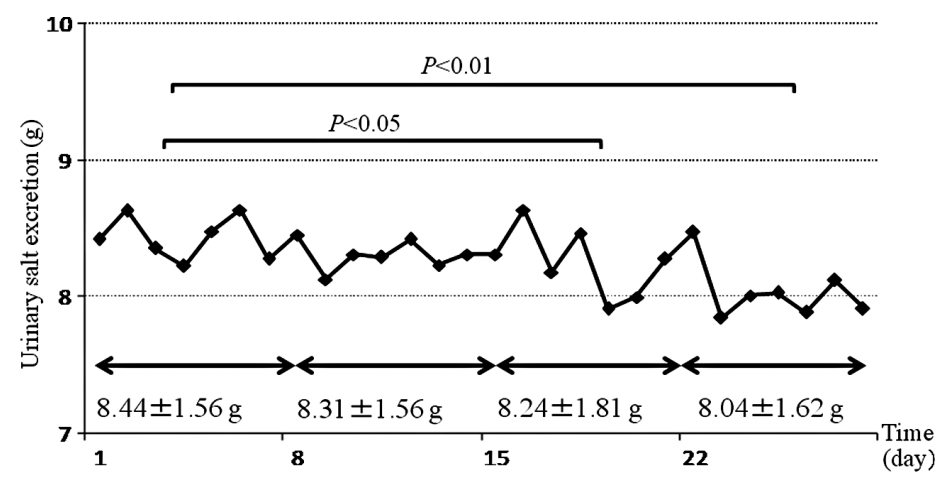

Figure 2. Trend of urinary salt excretion in all subjects. $\mathrm{P}<0.01 ; \mathrm{P}<0.05$ vs. mean salt excretion during the 1 st week.

Participant occupations included administrative officials $(\mathrm{n}=22)$ and public health nurses $(\mathrm{n}=8)$.

The intervention schedule is shown in Fig. 1. At baseline and 8 weeks later we measured blood pressure (BP), anthropometric variables and nutritional variables (by a dietary habits questionnaire). BP was measured with a sphygmomanometer by a public health nurse while subjects were sitting quietly at a room temperature of $25^{\circ} \mathrm{C}$. Anthropometric measurements, including waist circumference, were prformed by a dietitian. Survey of nutritional variables was carried out using a brief self-administered dietary history questionnaire (BDHQ) that was able to evaluate the nutrient intake of the previous month (11). The BDHQ was developed based on a self-administered dietary history questionnaire (DHQ) $(12,13)$. The practice of salt restriction was investigated using a salt restriction questionnaire. This included awareness of salt restriction and of the salt content of food, frequency of the use of seasoning, frequencies of eating pickles, noodles and soup, and opportunities to eat out. In this survey, the salt awareness was obtained based on the participant's self-evaluation. Scores for these items were added and the total score was used to investigate the relationship between awareness of salt restriction and actual urinary salt excretion. Subjects were asked to measure daily salt excretion at home for 4 weeks using the self-monitoring device.

Outcome markers. The main outcome measures were salt excretion levels and changes in systolic blood pressure (SBP) and diastolic blood pressure (DBP).
Statistical analysis. Values are presented as the means \pm standard deviation (SD). Differences in variables were compared by paired t-tests. A Chi-square test was also utilized when appropriate. Correlations between variables were analyzed using Pearson's product moment correlation coefficient and multiple linear regression analysis. All data were statistically analyzed with the assistance of the JMP software program (ver. 8; SAS Institute, Cary, NC, USA). The $\alpha$-level was set at $\mathrm{P}=0.05$.

\section{Results}

Baseline characteristics and urinary salt excretion. The mean age of the subjects was $43.2 \pm 8.8$ years. The mean salt excretion for all subjects (averaged over 4 weeks) was $8.05 \pm 1.61 \mathrm{~g} / \mathrm{day}$ and the range (maximum-minimum value) was $5.58 \pm 2.15 \mathrm{~g} /$ day in all subjects (Table I). Fig. 2 demonstrates the trend of daily salt excretion for all subjects throughout the 4 weeks. The average urinary salt excretion significantly decreased from week 1 to weeks 3 and $4(\mathrm{P}<0.05$ and $\mathrm{P}<0.01$, respectively).

Clinical parameters. Table II shows the changes in BP and anthropometric measures from baseline to the end of week 8 . The decrease in DBP was significant, but the decreases in SBP and in the anthropometric variables were not. Urinary salt excretion was significantly correlated with SBP $(r=0.54, \mathrm{P}=0.005)$, DBP $(r=0.55, \mathrm{P}=0.005)$, body 
Table I. Background of participants and urinary salt excretion.

\begin{tabular}{lc}
\hline Participants (no.) & 30 \\
(male/female) & $15 / 15$ \\
Age (years) & $43.20 \pm 8.8$ \\
Urinary salt excretion for 4 weeks (g/day) & $8.05 \pm 1.61$ \\
Maximal value (g/day) & $11.05 \pm 2.10$ \\
Minimum value (g/day) & $5.47 \pm 1.86$ \\
Range (g/day) & $5.58 \pm 2.15$ \\
Urinary salt excretion of 1 st week (g/day) & $8.44 \pm 1.56$ \\
Urinary salt excretion of $2 \mathrm{nd}$ week (g/day) & $8.31 \pm 1.56$ \\
Urinary salt excretion of 3rd week (g/day) & $8.24 \pm 1.81^{\mathrm{a}}$ \\
Urinary salt excretion of 4th week (g/day) & $8.04 \pm 1.62^{\mathrm{b}}$
\end{tabular}

Values are the means $\pm \mathrm{SD} .{ }^{\mathrm{a}} \mathrm{P}<0.05,{ }^{\mathrm{b}} \mathrm{P}<0.01$ vs. baseline by paired t-tests.

Table II. Changes in blood pressure, anthropometrics and nutritional intake.

\begin{tabular}{lcc}
\hline & Baseline & End of week 8 \\
\hline SBP (mmHg) & $123.0 \pm 20.7$ & $122.1 \pm 18.8$ \\
DBP (mmHg) & $77.7 \pm 14.3$ & $74.3 \pm 13.3$ \\
BW (kg) & $60.8 \pm 11.0$ & $60.6 \pm 11.3$ \\
BMI $\left(\mathrm{kg} / \mathrm{m}^{2}\right)$ & $22.8 \pm 3.3$ & $22.7 \pm 3.5$ \\
Waist circumference $(\mathrm{cm})$ & $81.1 \pm 9.1$ & $80.7 \pm 8.7$ \\
Total energy $(\mathrm{kJ})$ & $7,800 \pm 3,073$ & $7,155 \pm 2,801^{\mathrm{a}}$ \\
Protein $(\mathrm{g})$ & $62.3 \pm 21.2$ & $58.3 \pm 20.2$ \\
Fat $(\mathrm{g})$ & $53.8 \pm 21.0$ & $50.8 \pm 18.7$ \\
Carbohydrate $(\mathrm{g})$ & $245.2 \pm 90.7$ & $229.5 \pm 96.4$ \\
Potassium $(\mathrm{mg})$ & $2,269 \pm 905$ & $2,234 \pm 868$ \\
Calcium $(\mathrm{mg})$ & $416 \pm 180$ & $420 \pm 180$ \\
Magnesium $(\mathrm{mg})$ & $231 \pm 87$ & $221 \pm 82$ \\
Salt $(\mathrm{g})$ & $9.1 \pm 2.8$ & $8.5 \pm 3.1^{\mathrm{b}}$ \\
\hline
\end{tabular}

Values are the means $\pm \mathrm{SD} .{ }^{\mathrm{a}} \mathrm{P}<0.01 ;{ }^{\mathrm{b}} \mathrm{P}<0.05$ vs. baseline by paired t-test. SBP, systolic blood pressure; DBP, diastolic blood pressure; BW, body weight; BMI, body mass index.

weight $(\mathrm{BW})(\mathrm{r}=0.63, \mathrm{P}=0.005)$, body mass index $(\mathrm{r}=0.41$, $\mathrm{P}=0.05)$ and waist circumference (WC) $(\mathrm{r}=0.37, \mathrm{P}=0.05)$. Multiple linear regression analysis showed that DBP, body weight and WC were significantly and independently associated with urinary salt excretion $\left(R^{2}=0.5399\right.$ for total of DBP, BW and WC).

Dietary intake. Table II shows the changes in nutritional intake from baseline to the end of the 8th week. Energy and salt intakes decreased significantly, while intake of other nutritional variables did not significantly change during this period. Energy intake was significantly correlated with salt intake both at baseline $(\mathrm{r}=0.85, \mathrm{P}<0.0001)$ and at the end of the study $(r=0.90, P<0.0001)$. Furthermore, changes in both variables during the observation period were correlated with each other $(r=0.40, \mathrm{P}=0.0286)$.

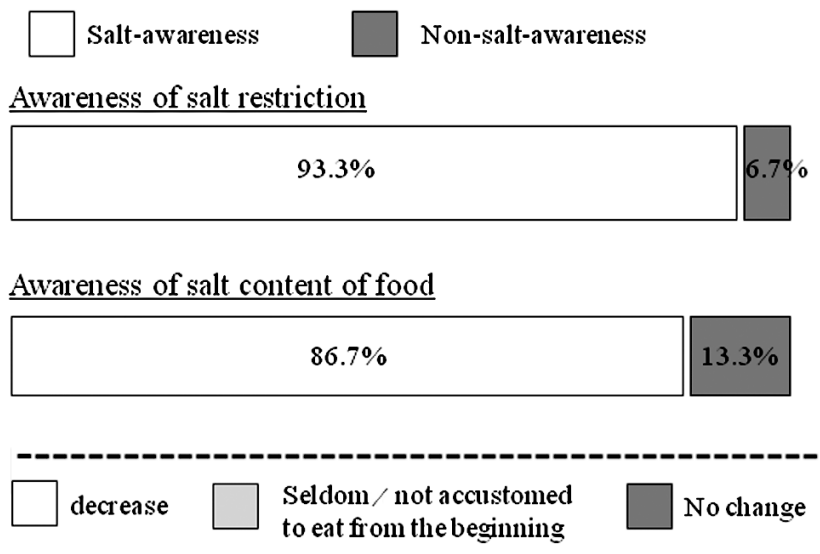

\section{Frequency of using seasoning}

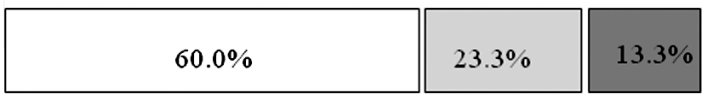

\section{Frequency of eating pickles}

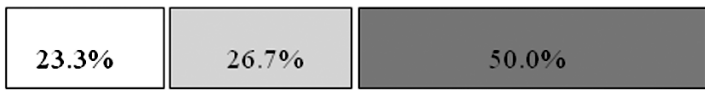

Frequency of eating soup

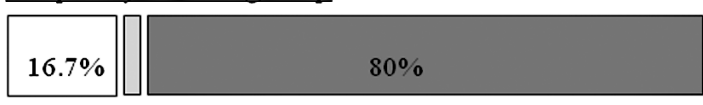

Opportunities to eat out

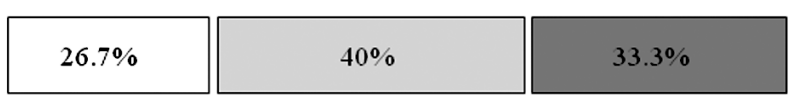

Frequency of eating noodles

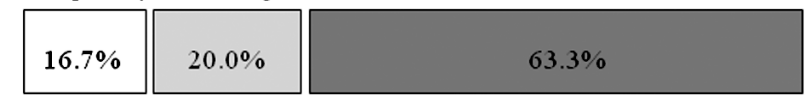

Figure 3. Changes in awareness of salt restriction during the study period.

Salt restriction questionnaire. The proportion of those who were aware of salt restriction increased from $47 \%$ at baseline to $90 \%$ after 8 weeks. Among the items investigated, awareness of the salt content of food increased and the use of seasoning decreased, while other items failed to show significant changes (Fig. 3). When urinary salt excretion was compared between the high- ( $>6$ points) and low-score ( $\leq 6$ points) groups, subjects in the high-score group showed significantly lower salt excretion $(7.07 \pm 0.80 \mathrm{~g} / \mathrm{day})$ compared to those in the low-score group $(8.46 \pm 1.70 \mathrm{~g} / \mathrm{day}, \mathrm{P}<0.01)$.

\section{Discussion}

There were two main findings of the present study. i) Selfmonitoring of urinary salt excretion at home for 4 weeks significantly reduced urinary salt excretion in healthy adults. ii) DBP, BW and WC were correlated with urinary salt excretion.

Ohta et al reported that urinary salt excretion is reduced by the use of a self-monitoring device by hypertensive patients, and that BW is significantly correlated with salt excretion (14). Meland et al also reported that during a randomized controlled trial for 12 weeks, BP decreased significantly in 
hypertensive patients who were using self-monitoring of urine for $\mathrm{Cl}^{-}$in addition to receiving nutritional instruction (15). These reports are consistent with our results showing the short-term usefulness of the self-monitoring of urinary salt excretion, and the relationship between salt excretion and BW. The present observation that self-monitoring of urinary salt excretion effectively decreases salt excretion even in healthy adults clearly indicates the potential usefulness of this method as an educational tool that will increase salt restriction and lead to the prevention of hypertension. However, salt restriction and weight control may not last long, as suggested by our TOHP II study (16). Thus, future studies are necessary to investigate long-term effectiveness of our salt-excretion selfmonitoring method for decreasing salt intake.

The decrease in salt intake was observed not only in those engaged in self-monitoring of urinary salt excretion, but also by results from our nutrition survey. In the nutrition survey, there was a significant and strong correlation between energy and salt intakes. Since salt intake of obese subjects was reported to be high compared to normal weight subjects (17), the restriction of energy intake may lead to salt reduction.

It is intriguing that self-monitoring of urinary salt excretion improved the awareness of salt restriction in normotensive subjects. However, there was no significant association between urinary salt excretion and awareness of salt restriction at baseline (data not shown). This observation is compatible with previous findings by Ohta et al that there is no relationship between awareness of salt restriction and the actual salt intake evaluated by 24-h urine collection (18). Since subjective awareness of salt restriction may not necessarily be reflected in actual salt reduction, monitoring of salt excretion seems extremely important to educate the population about the health benefits of salt restriction.

Concerning the validity of the self-monitoring device, Yamasue et al reported a significant correlation between i) salt excretion values estimated using overnight urine and a self-monitoring device, and ii) salt excretion measured by 24-h urine collection $(\mathrm{r}=0.72, \mathrm{P}<0.001)(10)$. Ohta et al also reported that salt excretion measured with a self-monitoring device correlated well with that determined by 24-h home urine evaluation in hypertensive subjects $(\mathrm{r}=0.63, \mathrm{P}<0.01)$ (14). Since the self-monitoring device can be used at home to evaluate daily urinary salt excretion, it can be used as a strong motivational tool to help individuals reduce salt intake. As a matter of fact, in this study urinary salt excretion by itself, without any specific educational program, reduced salt intake. Therefore, it seems more practical to use this device rather than 24-h urine collections for educating people about their need for salt restriction.

Several potential limitations of the present study include its non-randomized observational design, use of a small number of subjects and a short duration. Another limitation is that subjects were relatively young and of above average intelligence. Therefore, it is not yet clear whether the present findings can be extrapolated to the general population.

In conclusion, the use of a self-monitoring device leads to a decrease in salt excretion, which indicates that subjects reduced their salt intake. Use of the device thus seems to be an effective tool for educating healthy adults about the health benefits of salt restriction.

\section{Acknowledgements}

The present study was supported by funds from the Nishikyushu University. The authors greatly appreciate the technical assistance received from the staff of the Department of Health promotion, Tosu Health Center.

\section{References}

1. Intersalt: an international study of electrolyte excretion and blood pressure. Results for 24 hour urinary sodium and potassium excretion. Intersalt Cooperative Research Group. BMJ 297: 319-328, 1988.

2. Antonios TF and MacGregor GA: Salt intake: potential deleterious effects excluding blood pressure. J Hum Hypertens 9: $511-515,1995$.

3. Du Cailar G, Ribstein J and Mimran A: Dietary sodium and target organ damage in essential hypertension. Am J Hypertens 15: 222-229, 2002.

4. Espeland MA, Whelton PK, Kostis JB, et al: Predictors and mediators of successful long-term withdrawal from antihypertensive medications. TONE Cooperative Research Group. Trial of Nonpharmacologic Interventions in the Elderly. Arch Fam Med 8: 228-236, 1999.

5. Obarzanek E, Proschan MA, Vollmer WM, et al: Individual blood pressure responses to changes in salt intake: results from the DASH-Sodium trial. Hypertension 42: 459-467, 2003.

6. The INTERSALT Co-operative Research Group: appendix tables. Centre-specific results by age and sex. J Hum Hypertens 3: 331-407, 1989.

7. Nakagawa H, Morikawa Y, Okayama A, et al: Trends in blood pressure and urinary sodium and potassium excretion in Japan: reinvestigation in the 8th year after the Intersalt Study. J Hum Hypertens 13: 735-741, 1999.

8. Stamler J, Elliott P, Dennis B, et al: INTERMAP: background, aims, design, methods, and descriptive statistics (nondietary). J Hum Hypertens 17: 591-608, 2003.

9. Kawano Y, Tsuchihashi T, Matsuura H, Ando K, Fujita T and Ueshima H: Report of the Working Group for Dietary Salt Reduction of the Japanese Society of Hypertension: (2) assessment of salt intake in the management of hypertension. Hypertens Res 30: 887-893, 2007.

10. Yamasue K, Tochikubo O, Kono E and Maeda H: Selfmonitoring of home blood pressure with estimation of daily salt intake using a new electrical device. J Hum Hypertens 20: 593-598, 2006.

11. Sasaki S: Development and evaluation of dietary assessment methods using biomarkers and diet history questionnaire for individuals. In: Research for Evaluation Methods of Nutrition and Dietary Lifestyle Programs Held on Healthy Japan 21. Summary Report. Ministry of Health, Welfare and Labor, pp10-44, 2004.

12. Sasaki S, Ushio F, Amano K, et al: Serum biomarker-based validation of a self-administered diet history questionnaire for Japanese subjects. J Nutr Sci Vitaminol 46: 285-296, 2000.

13. Sasaki S, Yanagibori R and Amano K: Self-administered diet history questionnaire developed for health education: a relative validation of the test-version by comparison with 3-day diet record in women. J Epidemiol 8: 203-215, 1998.

14. Ohta Y, Tsuchihashi T, Miyata E and Onaka U: Usefulness of self-monitoring of urinary salt excretion in hypertensive patients. Clin Exp Hypertens 31: 690-697, 2009.

15. Meland E, Laerum E and Ulvik RJ: Salt restriction in hypertension - the effect of dietary advice and self monitoring of chloride concentration in urine. Scand J Clin Lab Invest 54: 399-404, 1994.

16. The Trials of Hypertension Prevention, phase II. The Trials of Hypertension Prevention Collaborative Research Group: Effects of weight loss and sodium reduction intervention on blood pressure and hypertension incidence in overweight people with high-normal blood pressure. Arch Intern Med 157: 657-667, 1997.

17. Frackiewicz J, Hamulka J, Wawrzyniak A and Gornicka M: [Students nutrients intake and risk of cardiovascular diseases]. Rocz Panstw Zakl Hig 60: 269-274, 2009.

18. Ohta Y, Tsuchihashi T, Ueno M, et al: Relationship between the awareness of salt restriction and the actual salt intake in hypertensive patients. Hypertens Res 27: 243-246, 2004. 